\title{
Necrosis retinal aguda: Una entidad de adultos en pediatría
}

\author{
Juan P. Torres Ty J. Pablo López G.
}

\section{Acute retinal necrosis: An adult disease visits pediatrics}

Acute retinal necrosis (ARN) is a serious condition that can impair vision. It mostly occurs in adult patients, especially those severely immunocompromised, in association with a reactivation of a herpes virus infection. Clinical and ophtalmological features of ARN and recommended diagnostic and management strategies are reviewed.

Key words: acute retinal necrosis, varicella-zoster virus, pediatrics.

Palabras claves: necrosis retinal aguda, virus varicela-zoster, pediatría.

\section{Introducción}

$\mathbf{L}$ a necrosis retinal aguda (NRA) fue descrita por primera vez en 1971, por Urayama y $\operatorname{cols}^{1}$ en Japón, en seis pacientes adultos que presentaban panuveítis, necrosis retinal periférica y desprendimiento de retina, siendo comunicada algunos años después, en E.U.A. y el Reino Unido ${ }^{2,3}$. En un comienzo, la etiología de la NRA era desconocida, hasta que en 1982, Culbertson y cols ${ }^{4}$, sugirieron el posible rol de los virus del grupo herpes como agentes causales, al ser demostrada su presencia en la histopatología, microscopia electrónica y cultivos virales de ojos enucleados de pacientes con NRA. Estudios posteriores fueron confirmando en el tiempo la asociación entre esta entidad y VVZ, VHS 1, VHS 2 y CMV CM-8. $^{5-}$

La mayoría de los casos ocurre en pacientes sobre 20 años de edad ( $>80 \%)$, con una mediana de 40 años?. En algunas ocasiones, los pacientes presentan un pródromo caracterizado por fiebre, cefalea y rigidez de cuello $^{10}$, y en las formas más clásicas, puede encontrarse dolor ocular leve a moderado, que se incrementa con los movimientos oculares, y ojo rojo. En relación con los síntomas visuales, es habitual que exista visión borrosa del ojo comprometido, "floaters" y disminución de la visión periférica. Menos frecuente es la pérdida grave de la visión central (como resultado del desprendimiento de retina en etapas más tardías de la enfermedad) y la pérdida visual aguda (resultante del compromiso del nervio óptico). Engstrom y $\operatorname{cols}^{11}$, en un estudio retrospectivo de 38 pacientes con NRA, encontraron visión borrosa en $54 \%$ de los casos, disminución del campo visual o pérdida de la visión periférica en $28 \%$, dolor ocular en 6 y $11 \%$ de los pacientes eran completamente asintomáticos.

La Sociedad Americana de la Uveítis publicó en
1994 los criterios diagnósticos de NRA ${ }^{12}$, que incluyen las siguientes observaciones clínicas:

- Áreas de necrosis retiniana focales, bien delimitadas en la periferia, más allá de la arcada vascular temporal.

- Rápida progresión de la necrosis, circunferencial, cuando no se instaura tratamiento antiviral.

- Evidencia de vasculopatía oclusiva con compromiso arteriolar.

- Reacción inflamatoria vítrea y de la cámara anterior.

Otras características, que no están dentro de estos criterios, incluyen la neuropatía óptica y la escleritis. En el examen oftalmoscópico de la cámara anterior, puede verse reacción celular leve a moderada, con precipitados queratínicos. La uveítis y la vitritis pueden ser tan graves, que dificultan el examen del polo posterior. La uveítis anterior progresa a panuveítis en el plazo de algunos días hasta una a dos semanas, avanzando a un compromiso que aumenta en 360 grados. La vasculopatía oclusiva y la vasculitis coroídea se asocia a la necrosis retiniana. Si bien la atrofia del nervio óptico o su inflamación es habitual en la NRA, no constituye un criterio diagnóstico de ésta. El borde entre las zonas de necrosis retiniana y zonas de retina sana es bien definido y geográfico. Las hemorragias retinianas, aunque ocasionalmente están presentes, no son un rasgo común. El daño en la retina, combinado con la tracción vítrea, conduce al desprendimiento de retina en 25 a $75 \%$ de los casos, en un tiempo promedio de 65 días luego de iniciada la enfermedad $^{10,13}$. El compromiso del ojo contralateral se describe en aproximadamente $33 \%$ de los casos (20 a $70 \%$ ) luego de cuatro a seis semanas de haber comenzado la NRA. En situaciones extremas, esto puede ocurrir hasta 20 años después ${ }^{14}$.
Hospital Luis Calvo Mackenna, Santiago, Chile

Departamento de Pediatría (JPTT) Servicio de Pediatría

Unidad de Oftalmología (JPLG) Hospital Del Salvador, Santiago, Chile Servicio de Oftalmología Departamento de Úvea (JPLG)

Recibido: 27 julio 2006 Aceptado: 11 abril 2007

Correspondencia a: Juan Pablo Torres Torretti jptorres@med.uchile.cl 


\section{Etiología de la necrosis retinal aguda}

La NRA esta dada, fundamentalmente, por la reactivación de un virus del grupo herpes, que puede ocurrir hasta años después de la primoinfección. Sin embargo, en pocos casos puede presentarse en relación con la primoinfección del VVZ. La NRA debida a VVZ y VHS 1 ocurre, predominantemente, en pacientes sobre 25 años de edad, mientras que VHS 2 en pacientes bajo ella ${ }^{9,15}$. Considerando todas las edades, el VVZ es dos veces más común que el VHS como causa de NRA.

Ganatra y cols ${ }^{9}$, estudiaron la etiología viral de la NRA en 28 pacientes (con un total de 30 ojos comprometidos). A través de RPC detectaron VVZ en 13 pacientes (15 ojos), VHS 1 en siete pacientes (7 ojos), VHS 2 en seis pacientes ( 6 ojos) y CMV en un paciente (1 ojo). En otro estudio realizado por Itoh y cols ${ }^{16}$ en Japón, de 16 pacientes con NRA, se detectó VVZ en nueve de ellos y VHS 2 en los siete restantes.

Abe y cols ${ }^{17}$, estudiaron pacientes con NRA producida por VVZ. Pudieron determinar que en aquellos pacientes en que se presenta una forma más fulminante de NRA, existe también una diversidad de virus con una capacidad de replicación mayor en el ojo afectado. Estos estudios fueron realizados con RPC anidada, amplificando las regiones variable R1 y R5 del VVZ. Por otro lado, Guex-Crosier y cols ${ }^{18}$, determinaron la importancia del estado inmunitario del paciente en relación con la gravedad de la NRA por VVZ. Aquellos individuos que presentaron una NRA leve o levemoderada, tenían una discreta alteración de la función inmune. En aquellos con mayor gravedad, se pudo determinar que existía una anomalía más acentuada de la función inmune (pacientes con SIDA, recuentos bajos de linfocitos T CD4, etc.).

Necrosis retinal aguda y biología molecular. El estudio de muestras de humor vítreo o humor acuoso mediante RPC alcanza una alta sensibilidad y especificidad en la identificación de los diferentes virus del grupo herpes en los casos de $\mathrm{NRA}^{19}$ y entrega un rápido informe. Tran y $\operatorname{cols}^{20}$, evaluaron el uso de la RPC en el diagnóstico etiológico de las NRA a partir de muestras de humor acuoso, en 22 pacientes con la enfermedad, logrando detección de agente etiológico en $86,4 \%$ de los casos. Resultados semejantes encontró Gargiulo y cols, en un estudio similar ${ }^{21}$.

Recientemente, Asano y cols ${ }^{22}$, demostraron una buena correlación entre carga viral (en humores vítreo y acuoso) y evolución clínica, empleando RPC en tiempo real y encontraron peor evolución en aquellos pacientes en que previamente se habían detectado cargas virales más altas.
Cabe cuestionarse si es posible detectar a través de RPC virus del grupo herpes en muestras de humor vítreo y acuoso de pacientes que no tienen NRA o que son sanos. Pendergast y $\operatorname{cols}^{23}$, estudiaron la prevalencia de ADN de virus grupo herpes en muestras de humores vítreo y acuoso, obtenidas de pacientes sanos que fueron sometidos a cirugía vitreoretinal. De los 75 pacientes analizados, ninguna de las muestras resultó positiva en la RPC, pese a existir entre 86 y $100 \%$ de prevalencia de anticuerpos contra virus de grupo herpes en estos pacientes.

La RPC realizada en muestras oculares permite ser de ayuda para iniciar un tratamiento oportuno y específico, de manera segura y menos invasora que los procedimientos de biopsia. Otros procedimientos diagnósticos, como serología, microscopia electrónica, cultivos virales, histopatología e inmunohistoquímica, pueden resultar más engorrosos, lentos o de mayor riesgo para el paciente.

\section{Comentarios terapéuticos en necrosis retinal aguda}

El objetivo central del tratamiento de la NRA es acelerar la resolución de la enfermedad en el ojo comprometido y prevenir el compromiso del ojo contralateral. No existen, a la fecha, ensayos clínicos prospectivos, aleatorios sobre el tratamiento de la NRA, siendo difícil la realización de éstos, dado el curso fulminante de la enfermedad.

El tratamiento de elección para la NRA es aciclovir $\left(500 \mathrm{mg} / \mathrm{m}^{2}\right.$ iv cada 8 horas durante 10 a 14 días, seguido de aciclovir oral $800 \mathrm{mg} /$ día durante 6 semanas adicionales), el cual posee buena acción antiviral sobre VHS y es efectivo contra VVZ. Si bien ganciclovir o foscarnet pueden tener mejor acción antiviral sobre CMV, este virus es causa inhabitual de NRA. El aciclovir ofrece, además, ventajas en su administración y seguridad respecto de los otros antivirales ${ }^{24}$. En un estudio retrospectivo ${ }^{25}$, sólo $12,9 \%$ de los pacientes tratados con aciclovir desarrollaron enfermedad en el ojo contralateral, comparado con $64,9 \%$ de los pacientes en el grupo control. Otras series han mostrado que las lesiones retinales tienen una resolución más rápida después del inicio del tratamiento con aciclovir, pero el tratamiento no parece disminuir el grado de inflamación (turbidez) del vítreo o prevenir el desprendimiento de retina secundario que frecuentemente complica esta enfermedad ${ }^{26,27}$. En casos de enfermedad grave o progresiva, puede considerarse el uso de ganciclovir o foscarnet.

En adición al aciclovir, los corticosteroides sistémicos (prednisona $0,5-1,0 \mathrm{mg} / \mathrm{kg} /$ día) pueden usarse 
después de 24 a 48 h de iniciar el tratamiento antiviral sistémico. La duración del tratamiento corticoesteroidal se basa en la respuesta clínica, principalmente por la turbidez del vítreo. Debido a la extensa vasculitis oclusiva en la NRA, algunos autores han sugerido el tratamiento con ácido acetilsalicílico. Esta terapia no ha sido analizada en un estudio clínico aleatorio ${ }^{28}$. Nuestro grupo (JPLG) prefiere utilizarla en la mayoría de los casos en que se observa un componente vasculítico oclusivo predominante. El tratamiento profiláctico con láser para demarcar la zona límite entre retina sana y enferma, preveniendo el desprendimiento de retina secundario, también ha sido utilizado en algunos ensayos con buena respuesta, pero la real utilidad de esta terapia también se ve afectada por la naturaleza retrospectiva de dichos estudios ${ }^{29}$. Otros autores han sugerido la vitrectomía profiláctica, el buckling escleral y el endoláser para ojos con alto riesgo de desprendimiento de retina. Pocos datos en la literatura apoyan estas terapias ${ }^{30}$.

La cirugía está indicada para reparar los desprendimientos de retina en pacientes con NRA. Estos desprendimientos son técnicamente muy difíciles de reparar y la devastadora vítreo retinopatia proliferativa (PVR), similar a la de los diabéticos complicados, es común en este tipo de desprendimientos, lo que ensombrece aún más el pronóstico de la enfermedad ${ }^{30}$. Aún menos estudiado ha sido el efecto del tratamiento en la neuropatía óptica que suele acompañar a la NRA. Los corticoesteroides y fenestración del nervio óptico se han intentado con fines terapéuticos, pero a la fecha no existen datos concluyentes provenientes de estudios controlados respecto de este tema como para sugerir estas modalidades terapéuticas, no exentas de complicaciones, en forma rutinaria.

Finalmente, aunque aciclovir y los corticoesteroides se usan constantemente para tratar pacientes con NRA, aún no hay estudios aleatorios y de doble ciego que demuestren en forma definitiva un beneficio de este tratamiento.

La NRA es una enfermedad infrecuente y de pre-

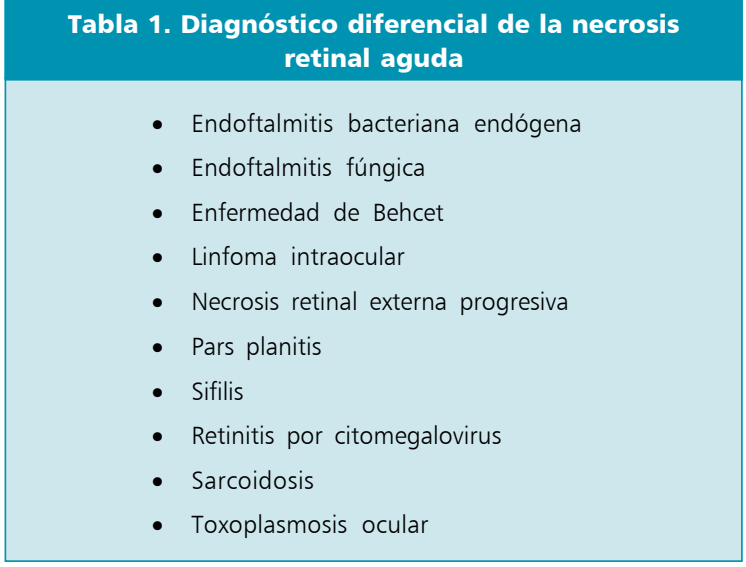

sentación muy baja en la práctica oftalmológica diaria, pero la infección con los diversos virus del grupo herpes es muy común en la población general. La susceptibilidad de algunos individuos para desarrollar la NRA persiste como uno de los más grandes enigmas de esta enfermedad. Varias patologías infecciosas e inflamatorias pueden presentarse en forma similar a la NRA (Tabla 1). Un oftalmólogo experimentado en el diagnóstico y tratamiento de estas enfermedades de infrecuente presentación así como un alto índice de sospecha y el trabajo en equipo con infectólogos son sin duda los elementos más importantes en el manejo de estas entidades.

\section{Resumen}

La necrosis retinal aguda (NRA) es una afección grave que amenaza la visión. Se presenta en adultos, en especial aquellos profundamente inmunocomprometidos, como consecuencia de la reactivación de virus del grupo herpes. Se revisan la entidad clínica de la NRA, su diagnóstico nosológico y etiológico, como el manejo recomendado actualmente.

\section{Referencias}

1.- Urayama A, Yamada N, Sasaki T, et al. Unilateral acute uveitis with retinal periarteritis and detachment. Jpn J Clin Ophthalmol 1971; 25: 607-19.

2.- Willerson D Jr, Aaberg T M, Reeser F H. Necrotizing vaso-occlusive retinitis. Am J Ophthalmol 1977; 84: 209-19.

3.- Young NJA, Bird AC. Bilateral acute retinal necrosis. Br J Ophthalmol 1978; 62: 581-90.
4.- Culbertson W W, Blumenkranz M S, Haines H. The acute retinal necrosis syndrome: histopathology and etiology. Ophthalmology 1982; 89: 1317-25.

5.- Freeman W R, Thomas E L, Rao N A. Demonstration of herpes group virus in acute retinal necrosis syndrome. Am J Ophthalmol 1986; 102: 701-9.

6.- Rungger-Brandle E, Roux L, Leuenberger P M. Bilateral acute retinal necrosis. Ophthalmology 1984; 91: 1648-58.
7.- Culbertson W W, Blumenkranz M S, Pepose J S. Varicella zoster virus is a cause of the acute retinal necrosis syndrome. Ophthalmology 1986; 93: 559-69.

8.- Pepose J S, Flowers B, Stewart J A. Herpesvirus antibody levels in the etiological diagnosis of the acute retinal necrosis syndrome. Am J Ophthalmol 1992; 113: 248-56.

9.- Fisher J P, Lewis M L, Blumenkranz M S. The acute retinal necrosis syndrome: 
Clinical manifestations. Ophthalmology 1982; 89: 1309-16.

10.- Clarkson J G, Blumenkranz M S, Culbertson W W. Retinal dettachment following the acute retinal necrosis syndrome. Ophthalmology 1984; 91: 1665-68.

11.- Engstrom R E Jr, Holland G N, Margolis T P. The progressive outer retinal necrosis syndrome: a variant of necrotizing herpetic retinopathy in patients with AIDS. Ophthalmol 1994; 101: 1488-1502.

12.- Holland G N and the Executive Committee of the American Uveitis Society: Standard diagnostic criteria for the acute retinal necrosis syndrome. Am J Ophthalmol 1994; 117: 663-7.

13.- Schlingemann RO, Bruineneberg $M$, Wertheim-van Dillen P, Feron E. Twenty years' delay of contralateral eye involvement in herpes simplex virus type 2-associated bilateral acute retinal necrosis syndrome. Am J Ophthalmol 1996; 122: 891-2.

14.- Van Gelder R N, Willig J L, Holland G N, Kaplan H J. Herpes simplex virus type 2 as a cause of acute retinal necrosis syndrome in young patients. Ophthalmology 2001; 108: 869-76.

15.- Ganatra J B, Chandler D, Santos C, Kupperman B, Margolis T P. Viral causes of the acute retinal necrosis syndrome. Am J Ophthalmol 2000; 129: 166-72. 16.- Itoh N, Matsumura N, Ogi A. High prevalence of herpes simplex virus type 2 in acute retinal necrosis syndrome associated with herpes simplex virus in Japan. Am J Ophthalmol 2000; 129: 404-5.

17.- Abe T, Sato M, Tamai M. Variable R1 region in varicella-zoster virus in fulminant type of acute retinal necrosis syndrome. Br J Ophthalmol 2000; 84: 193-8.

18.- Guex-Crosier Y, Rochat C, Herbort C P. Necrotizing herpetic retinopathies: a spectrum of herpes virus-induced diseases determined by the immune state of the host J Oc Immunol Inflamm 1997; 5: 259-65.

19.- Knox C M, Chandler D, Short J A. Polymerase chain reaction-based assays of vitreous samples for the diagnosis of viral retinitis. Ophthalmology 1998; 105: 37-45.

20.- Tran T H, Rozenberg F, Cassoux N, Rao N, LeHoang P, Bodaghi B. Polymerase chain reaction analysis of aqueous humor samples in necrotising retinitis. $\mathrm{Br} \mathrm{J}$ Ophthalmol 2003; 87: 79-83.

21.- Gargiulo F, De Francesco M A, Nascimbeni G, Turano R. Polymerase chain reaction as a rapid diagnostic tool for therapy of acute retinal necrosis syndrome. J Med Virol 2003; 69: 397-400.

22.- Asano S, Yoshikawa T, Kimura H, Enomoto Y, Ohashi M. Monitoring herpesvirus DNA in three cases of acute retinal necrosis by real-time PCR. J Clin Virol 2004; 29: 206-9.

23.- Pendergast S D, Werner J, Drevon A. Absence of herpesvirus DNA by polymerase chain reaction in ocular fluids obtained from immunocompetent patients. Retina 2000;20: 389-93.

24.- Nussenblat R, Withcup S. Uveitis: Fundamentals and Clinical Practice. Third edition, Elsevier (USA), 2004.

25.- Palay D A, Sternberg P Jr, Davis J, Lewis H, Holland G N, Mieler W F, et al. Decrease in the risk of bilateral acute retinal necrosis by acyclovir therapy. Am J Ophthalmol 1991;112: 250-5.

26.- Blumenkranz M S, Culbertson W W, Clarkson J G, Dix R. Treatment of the acute retinal necrosis syndrome with intravenous acyclovir. Ophthalmology 1986; 93: 296-300.

27.- Matsuo T, Nakayama T, Koyama T, Koyama M, Matsuo N. A proposed mild type of acute retinal necrosis syndrome. Am J Ophthalmol 1988; 105: 579-83.

28.- Ando F, Kato M, Goto S, Kobayashi K, Ichikawa H, Kamiya T. Platelet function in bilateral acute retinal necrosis. Am J Ophthalmol 1983; 96: 27-32.

29.- Sternberg P Jr, Han D P, Yeo J H, Barr C C, Lewis H, Williams G A, et al. Photocoagulation to prevent retinal detachment in acute retinal necrosis. Ophthalmology 1988; 95: 1389-93.

30.- Blumenkranz M, Clarkson J, Culbertson W W, Flynn H W, Lewis M L, Young G A. Vitrectomy for retinal detachment associated with acute retinal necrosis. Am J Ophthalmol 1988; 106 (4): 426-9. 